\title{
An Audit into the Screening Tests used for Cushing's syndrome
}

\section{Mark Platt, Elizabeth Robinson, David Ray. \\ Centre for Endocrinology and Diabetes, \\ University of Manchester.}

\section{Background}

We audited the use of screening tests for Cushing syndrome (CS), used by Manchester Royal Infirmary in 2012. This was against guidelines provided by The Endocrine Society and MRI hospital policy.

\section{Objectives of the Audit}

Specifically, the objectives of the audit were to investigate:-

- Were patients screened for CS adequately,

- Were discordant results followed up,

- Could medication have interfered with the result,

- Were positive results managed properly.

This audit also determined how well the tests administered correlated with each other as a means to assess their consistency in screening for CS.

\section{Screening tests for CS}

Urinary Free Cortisol (UFC)

- This test has a sensitivity of $45-71 \%$ and a specificity of $91 \%$. $[1,2]$

Overnight Dexamethosone Suppression test (oDST)

- This test has a sensitivity $85-95 \%$ of and a specificity of $80-95 \%$. [1,4]

Late Night Salivary Cortisol (LNSC)

- This test has a sensitivity of $92 \%$ and a specificity of $96 \%$. [1,3]

\section{Criteria used for CS screening audit}

The criteria for this audit were:

- Patients should have at least 2 UFCs performed or $1 \mathrm{oDST}$.

- Patients should not have a random plasma cortisol to screen for CS as this is insufficient.

- Patients with positive results were followed up appropriately.

- Patients with discordant results were investigated further.

- The medication prescribed did not have an impact on the test results.

- The different tests provided by MRI should produce consistent results with each other.

These criteria are based on the guidelines of the Endocrine Society. In particular, the criteria verify that patients suspected of having CS should have at least 2 UFCs performed or $1 \mathrm{oDST}$. Unfortunately, data for LNSC was not available in 2012 as the test was not in routine use in our clinical service; it is now. [1]

\section{Data Collection Methodology}

- The data for all the screenings tests used to investigate cortisol levels in 2012 was collected from the Clinical Biochemistry Dept at MRI.

- The test values were evaluated to determine whether the test result was positive or negative.

- Medisec records were used to ascertain the reason why the test was done and the depth of suspicion of CS.

- Medisec records were also used to determine the medication patients were taking at the time of the test.

- Patients were excluded from the audit if the above data was not complete or if they were obvious medical outliers.

\section{Results}

- 80 patients had screening tests for CS in 2012 and, overall, the management of these patients satisfied the audit criteria in all cases.

- 11 Patients had positive UFC or oDST results. They were followed up with further screening; appropriate treatment or clinical judgement was used to stop further, unnecessary investigations. The management of these patients is summarised in Figure 1.

- Some patients had only one UFC test performed to screen for CS, but none of these patients had a strong suspicion of CS.
Management of Positive CS screening tests

Figure 1
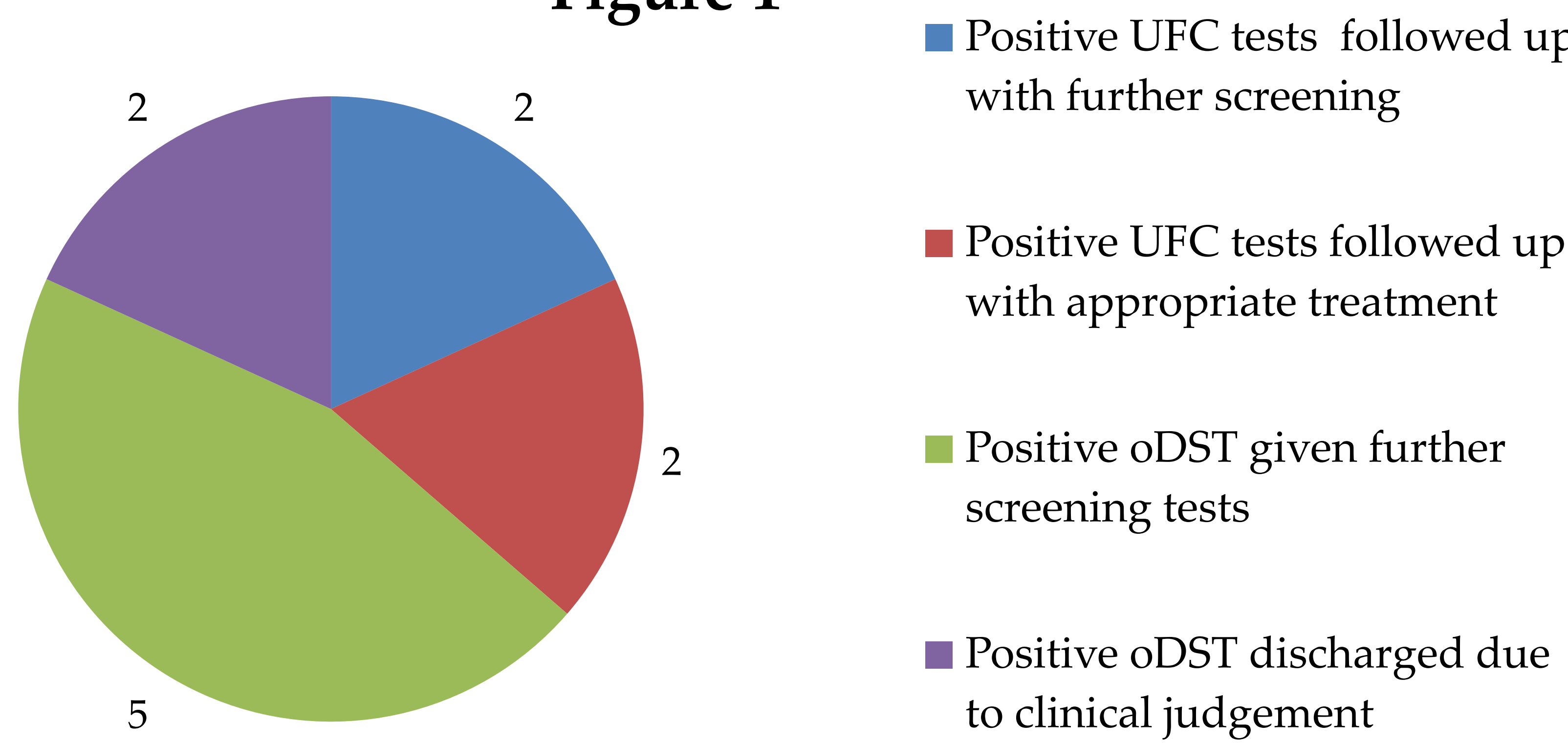

- In 16 cases, the patients were on medications that influenced the screening test results.

- In 14 of the patients, this was taken into account or the test result was unlikely to have been changed by that drug.

- In one case, the patient was on multiple drugs that suppress cortisol secretion. Another patient was on a drug that significantly reduces cortisol secretion. In both cases, the patient's medication that could have caused the negative result was not taken into account.

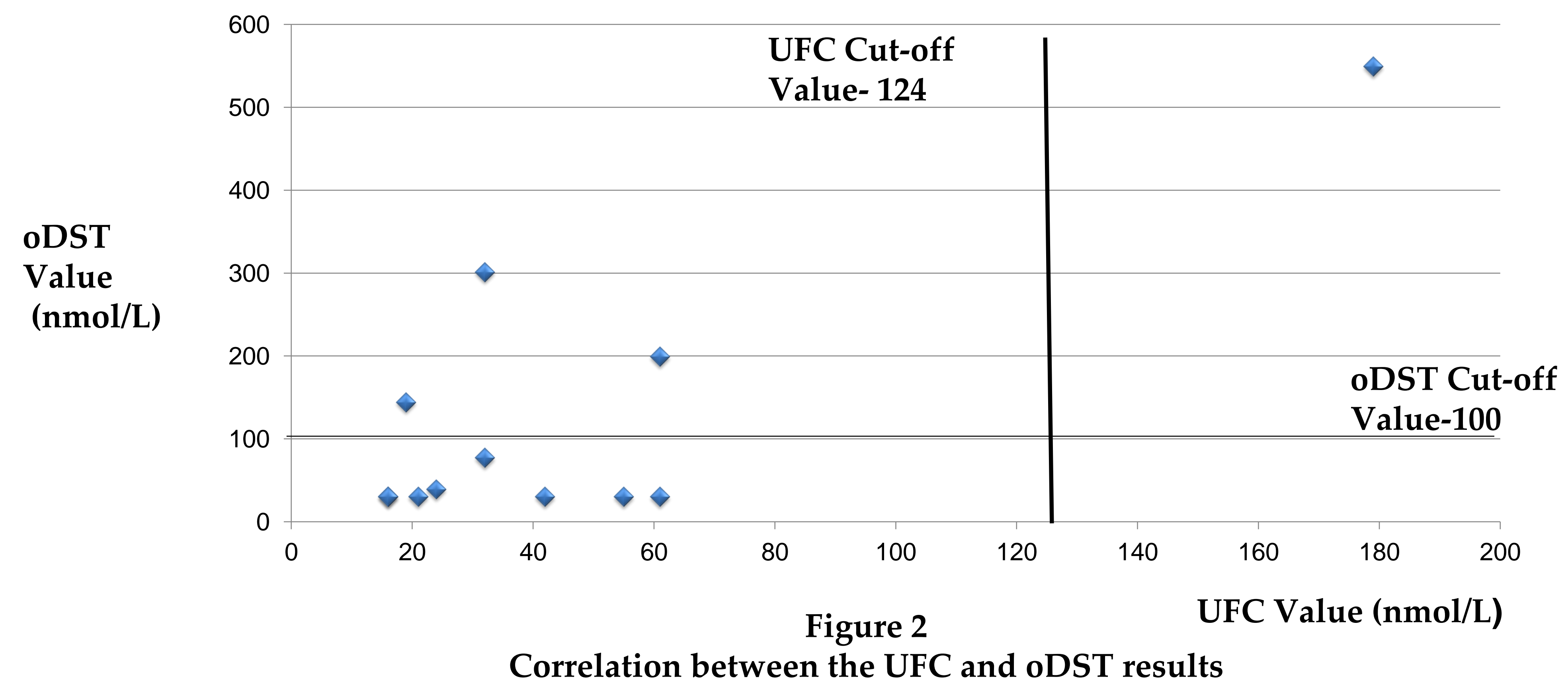

The correlation between UFC and oDST, shown in Figure 2 above, is weak. SPSS was used to calculate the Kappa co-efficient which was 0.308 . This was not significant. To examine this unexpectedly poor correlation, a larger number of patient samples would be necessary.

\section{Conclusions}

1. The screening tests used for CS were performed adequately in 2012. The screening tests themselves used did not correlate with each other strongly and the effects of medication on the screening tests were not always taken into account.

2. To improve the quality of care a new set of guidelines for screening for CS, highlighting the importance of medication, should be instated within the trust. Additionally, the LNSC screening test should be recommended due to its greater sensitivity.

3. Re-auditing to see how the screening for CS has changed should be done in the future. Particular attention should be given to how well the UFC, oDST and LNSC agree with each other.

\section{References}

[1] Nieman, I.N. et al. The Diagnosis of Cushing's Syndrome: An Endocrine Society Clinical Pracitce Guideline. Journal of Clinical Endocrinology \& Metabolism 2008. 93(5); 1526-1540. [2] Alexandraki, K.I, Grossman, A.B. Is urinary free cortisol of value in the diagnosis of Cushing's Syndrome and Adrenal Insufficiency. J Clin Endocrinol Metab, 2009. 94(10):3647-3655. [3] Raff, H. Cushing's syndrome: diagnosis and surveillance using salivary cortisol. Pituitary 2012 $15: 64-70$

4] Tisgos, C. Chrousos, G P. Differential Dagnosis and Management of Cushing's Syndrome. Annu. Rev Med. 1996. 47:443-61. 